MATHEMATICS OF COMPUTATION

Volume 76, Number 258, April 2007, Pages 777-793

S 0025-5718(06)01942-9

Article electronically published on December 13, 2006

\title{
DISCRETISATION OF AN INFINITE DELAY EQUATION
}

\author{
T. SENGADIR
}

\begin{abstract}
In this paper, a Banach phase space containing $\mathbf{B C}(-\infty, 0]$ and contained in $\mathbf{C}(-\infty, 0]$ is defined with which existence of a solution and convergence of a discrete scheme are proved for an infinite delay differential equation.
\end{abstract}

\section{INTRODUCTION AND PRELIMINARIES}

In this paper we prove the existence of a solution and convergence of a discrete scheme for the following functional differential equations with infinite delay.

$$
\begin{aligned}
& x^{\prime}(t)=a x(t)+\sum_{k=1}^{\infty} b_{k} x\left(t-\tau_{k}\right), \quad t \geq 0, \\
& x(\theta)=\phi(\theta), \quad \theta \in(-\infty, 0] .
\end{aligned}
$$

Here $a$ is a non-zero real, $\mathbf{b}=\left\{b_{k}\right\}_{k=1}^{\infty} \in l^{1}$, with $b_{k} \neq 0$ for all $k \geq 1,\left\{\tau_{k}\right\}_{k=1}^{\infty}$ is a strictly increasing sequence of strictly positive reals such that $\lim _{k \rightarrow \infty} \tau_{k}=\infty$ and $\phi:(-\infty, 0] \longrightarrow \mathbb{R}$ is continuous.

By standard arguments, it can be shown that the solution to this problem gives rise to a semigroup on $\mathbf{B U C}(-\infty, 0]$ whose generator is the derivative operator. If this derivative is replaced by a finite difference, unlike in the case of a finite delay equation, we get an infinite system of first order differential equations. To get a finite system of discretised equations, we can confine ourselves to a finite interval at each stage of computation. But, consider $u \in \mathbf{B U C}(-\infty, 0]$ and the sequence $\left\{u_{n}\right\}$ of functions defined as

$$
\begin{aligned}
u_{n}(x) & =u(x), x \in[-n, 0], \\
& =u(-n) x \in(-\infty,-n] .
\end{aligned}
$$

Now, we can easily see that, in general, $\lim _{n \rightarrow \infty}\left\|u_{n}-u\right\|_{\infty} \neq 0$. So, approximation schemes which use the initial data restricted to finite intervals at every stage may not converge. In this paper, we define a phase space which enables us to prove a convergence result for a finite difference scheme. This scheme uses the initial data confined to a finite interval at every stage of computation.

It is well known that unlike in the case of finite delays, the choice of a phase space for the infinite delay equation is a difficult one. [1, 3, 4, 5, and 2, are some of the references in this direction. For a Frechét space approach refer to [7].

Received by the editor April 5, 2005 and, in revised form, March 31, 2006.

2000 Mathematics Subject Classification. Primary 34K28.

Key words and phrases. Functional differential equations, infinite delay, numerical solutions.

The author would like to thank the Management Committee of SSNCE for their constant encouragement, support, and for setting up computational lab.

(C)2006 American Mathematical Society Reverts to public domain 28 years from publication 
If $x$ is the solution of a delay differential equation, then the function $\varphi_{t}$ defined as $\varphi_{t}(\theta)=x(t+\theta)$ is considered as the state of the system at $t$. In general, $\varphi_{t}$ is an element of an infinite dimensional Banach space and its finite dimensional approximations are studied. These finite dimensional approximations are solutions of a system of differential equations. For example, refer to [9]. In [10, the finite dimensional approximations belong to spline functions.

Approximation of infinite delay equations are examined in 11. For detailed references, see [12].

Our results on discretisation are based on Theorem 1.7 which is a version of the Trotter-Kato theorem proved in [6]. The following definitions and results in the theory of semigroups will be needed. [8] is a standard reference in this context.

Definition 1.1. Let $(X,\|\|)$ be a Banach space. A one parameter family of bounded linear maps $T_{t}: X \rightarrow X, t \in[0, \infty)$ is said to be a strongly continuous semigroup of bounded linear operators on $X$ if

(i) $T_{0}=I$,

(ii) $T_{t+s}=T_{t} T_{s}$ for every $t, s \geq 0$,

(iii) $\lim _{t \rightarrow 0}\left\|T_{t} x-x\right\|=0, \quad x \in X$

Definition 1.2. A semigroup of bounded linear operators on $X$ is said to be of class $G(M, \omega, X)$ if there exist constants $M \geq 1$ and $\omega \in R$ such that $\left\|T_{t}\right\| \leq$ $M e^{\omega t}$. A strongly continuous semigroup of class $G(1,0, X)$ is called a semigroup of contractions.

Definition 1.3. Let the linear operator $A$ be defined as follows:

$$
D(A)=\left\{x \in X: \lim _{h \rightarrow 0^{+}} \frac{T_{h} x-x}{h} \text { exists }\right\} .
$$

For $x \in D(A), A x$ is defined as

$$
A x=\lim _{h \rightarrow 0^{+}} \frac{T_{h} x-x}{h} .
$$

We say that $A$ generates the semigroup $T_{t}$ or that $A$ is the infinitesimal generator of $T_{t}$.

Theorem 1.4. If $T_{t}$ is a semigroup of bounded linear operators, then there are constants $M \geq 1$ and $w \in R$ such that $T$ is of the class $G(M, \omega, X)$. Further, its infinitesimal generator $A$ is a closed and densely defined linear operator which is unique.

Besides, if $B: X \longrightarrow X$ is a bounded linear operator, then it generates a semigroup which is given by $T_{t}=e^{t B}$.

Definition 1.5. Let $X$ be a real Banach space and $X^{*}$ its dual. For $x \in X$, $F(x) \subseteq X^{*}$ is defined as

$$
F(x)=\left\{\eta \in X^{*}:\langle x, \eta\rangle=\|x\|^{2}=\|\eta\|^{2}\right\} .
$$

A closed operator $A$ with a dense domain $D(A)$ is said to be dissipative if for every $x \in D(A)$, there is $\eta \in F(x)$ such that $\langle A x, \eta\rangle \leq 0$.

Theorem 1.6 (Lumer-Philips). If $A$ is dissipative and there is $\lambda_{0}>0$ such that the range $R\left(\lambda_{0} I-A\right)$ of $\lambda_{0} I-A$ is $X$, then $A$ is the infinitesimal generator of a strongly continuous semigroup of contractions. 
The following version of Trotter-Kato approximation is proved in 6 .

Let $X$ and $X_{n}$ be a Banach spaces with norms $\|\cdot\|$ and \|\|$_{n}$. For every $n=$ $1,2, \ldots$, there exist bounded linear operators $P_{n}: X \longrightarrow X_{n}$ and $E_{n}: X_{n} \longrightarrow X$ satisfying the following:

(1) $\left\|P_{n}\right\| \leq M_{1},\left\|E_{n}\right\|_{n} \leq M_{2}$ where $M_{1}$ and $M_{2}$ are independent of $n$;

(2) $\left\|E_{n} P_{n} x-x\right\| \longrightarrow 0$ as $n \rightarrow \infty$ for all $x \in X$;

(3) $P_{n} E_{n}=I_{n}$ where $I_{n}$ is the identity operator on $X_{n}$.

Theorem 1.7. Let $A: D(A) \longrightarrow X$ be a closed and densely defined operator in the class $G(M, \omega, X)$ and $A_{n}: X_{n} \longrightarrow X_{n}$ be bounded linear maps in $G\left(M, \omega, X_{n}\right)$. Let the semi-groups generated by $A$ and $A_{n}$ be denoted by $T_{t}$ and $T_{t}^{n}$. Then the following are equivalent:

(a) For all $u \in D(A)$ there exists a sequence $\bar{u}_{n} \in X_{n}$ such that

$$
\lim _{n \rightarrow \infty} E_{n} \bar{u}_{n}=u \text { and } \lim _{n \rightarrow \infty} E_{n} A_{n} \bar{u}_{n}=A u .
$$

(b) $\lim _{n \rightarrow \infty}\left\|E_{n} T_{t}^{n} P_{n} x-T_{t} x\right\| \rightarrow 0$ as $n \rightarrow \infty$ for all $x \in X$.

We require the following definitions and results in the subsequent sections:

Let $J \subset \mathbb{R}$ be a non-compact interval and let $J_{k}$ be an increasing sequence of compact intervals such that $\bigcup_{n=1}^{\infty} J_{k}=J$. Let $\mathbf{b}=\left\{b_{k}\right\} \in l^{1}$. For $u \in \mathbf{C}(J)$, define $p_{k}(u)=\sup _{x \in J_{k}}|u(x)| . C(J)$ is a Frechét space whose topology is given by the family of semi-norms $\left\{p_{k}: k \in \mathbb{N}\right\}$. We shall define a Banach space whose norm is given by a "linear combination" of these semi-norms. As a consequence of our assumption that $\left\{b_{k}\right\} \in l^{1}, \mathbf{B U C}(J)$ is contained in the new Banach space. Its topology is weaker than that of $\mathbf{B U C}(J)$ and stronger than that of $\mathbf{C}(J)$.

Definition 1.8. Let $J_{k}$ and the sequence $\left\{b_{k}\right\}$ be as above. Define $\mathbf{C}_{\sigma}(J)$ as

$$
\mathbf{C}_{\sigma}(J)=\left\{u \in \mathbf{C}(J): \sum_{k=1}^{\infty}\left|b_{k}\right| p_{k}(u)<\infty\right\} .
$$

Further, \|\|$_{\sigma}: \mathbf{C}(J) \rightarrow \mathbb{R}^{+}$is defined as $\|u\|_{\sigma}=\sum_{k=1}^{\infty}\left|b_{k}\right| p_{k}(u)$.

Proposition 1.9. \|\|$_{\sigma}$ is a norm on the space $\mathbf{C}_{\sigma}(J)$ and with respect to this norm, $\mathbf{C}_{\sigma}(J)$ is a Banach space. The space $\mathbf{B U C}(J)$ is continuously embedded in $\mathbf{C}_{\sigma}(J)$. Further, we have the following criterion for convergence in this Banach space:

Let $u_{n}$ be a sequence of functions in $\mathbf{C}_{\sigma}(J)$ and $u \in \mathbf{C}_{\sigma}(J)$. Assume the following:

(i) For a fixed $k$,

$$
\lim _{n \rightarrow \infty} p_{k}\left(u_{n}-u\right)=0
$$

(ii) There exists a sequence $\left\{\alpha_{k}\right\}$ of non-negative reals with $\sum_{k=1}^{\infty}\left|b_{k}\right| \alpha_{k}<\infty$ with

$$
p_{k}\left(u_{n}-u\right) \leq \alpha_{k}
$$

for all $n$ and $k$.

Then,

$$
\lim _{n \rightarrow \infty} u_{n}=u
$$


Proof. We only prove the convergence criterion. The other assertions are easy to see:

$$
\sum_{k=1}^{\infty}\left|b_{k}\right| p_{k}\left(u_{n}-u\right)=\sum_{k=1}^{N}\left|b_{k}\right| p_{k}\left(u_{n}-u\right)+\sum_{k=N+1}^{\infty}\left|b_{k}\right| p_{k}\left(u_{n}-u\right) .
$$

For a fixed $N$, the limit of the finite sum as $k \rightarrow \infty$ is zero by (i). The limit of the infinite sum is also zero because of (ii) and $\sum_{k=1}^{\infty}\left|b_{k}\right| \alpha_{k}<\infty$.

Let $n \in \mathbb{N}$. Consider the finite dimensional vector space $\mathbb{R}^{n^{2}+1}$. We define the norm $\|\cdot\|_{n}$ on $\mathbb{R}^{n^{2}+1}$ as follows:

$$
\|v\|_{n}=\sum_{k=1}^{n}\left|b_{k}\right| \max \left\{\left|v_{0}\right|,\left|v_{1}\right|, \ldots,\left|v_{k n}\right|\right\}+\sum_{k=n+1}^{\infty}\left|b_{k}\right| \max \left\{\left|v_{0}\right|,\left|v_{1}\right|, \ldots,\left|v_{n^{2}}\right|\right\}
$$

Here $v=\left(v_{0}, v_{1}, \ldots, v_{n}, v_{n+1}, v_{n+2}, \ldots, v_{2 n}, \ldots, v_{n^{2}}\right)$.

\section{A PHASE SPACE FOR THE INFINITE DELAY EQUATION}

Let $a,\left\{b_{k}\right\}$, and $\tau_{k}$ be as in the previous section. Let $[x]$ be the largest integer less than or equal to $x$. Define $m_{1}=\left[-\tau_{1}\right]$ and $m_{k}$ as $\left[-\tau_{i}\right]$ where $i$ is the smallest positive integer such that $-\tau_{i}<m_{k-1}$. It is clear that $m_{k}$ is a strictly decreasing sequence of negative integers and $-\tau_{k} \in\left[-m_{k}, 0\right]$.

The space $C_{\sigma}(-\infty, 0]$ is defined as

$$
C_{\sigma}(-\infty, 0]=\left\{\phi \in \mathbf{C}(-\infty, 0]: \sum_{k=1}^{\infty}\left|b_{k}\right| \sup _{\theta \in\left[m_{k}, 0\right]}|\phi(\theta)|<\infty\right\} .
$$

The proof of the next proposition follows from Proposition 1.9.

Proposition 2.1. For $\phi \in \mathbf{C}_{\sigma}(-\infty, 0]$ define

$$
\|\phi\|_{\sigma} a s\|\phi\|_{\sigma}=\sum_{k=1}^{\infty}\left|b_{k}\right| \sup _{\theta \in\left[m_{k}, 0\right]}|\phi(\theta)| .
$$

Then \|\|$_{\sigma}$ is a norm on $\mathbf{C}_{\sigma}(-\infty, 0]$ and $\mathbf{C}_{\sigma}(-\infty, 0]$ is a Banach space.

Next we define a linear operator $A$ as follows: Let

$$
D(A)=\left\{\phi \in \mathbf{C}^{\mathbf{1}}(-\infty, 0]: \phi, \phi^{\prime} \in \mathbf{C}_{\sigma}(-\infty, 0] \text { and } \phi^{\prime}(0)=L \phi\right\}
$$

where $L: \mathbf{C}_{\sigma}(-\infty, 0] \rightarrow \mathbb{R}$ as $L \phi=a \phi(0)+\sum_{k=1}^{\infty} b_{k} \phi\left(-\tau_{k}\right)$ for $\phi \in D(A), A \phi=\phi^{\prime}$.

Theorem 2.2. The operator $A$ defined above generates a strongly continuous semigroup $\left\{T_{t}: t \geq 0\right\}$ of bounded linear operators on $\mathbf{C}_{\sigma}(-\infty, 0]$. Further, for a given $\phi \in \mathbf{C}_{\sigma}(-\infty, 0]$, the map $x: \mathbb{R} \longrightarrow \mathbb{R}$ defined as

$$
\begin{aligned}
& x(t)=\phi(t), t \in(-\infty, 0], \\
& x(t)=\left[T_{t} \phi\right](0) t \in(0, \infty),
\end{aligned}
$$

is a unique solution to (1.1).

Remark. If we can show directly that $A$ generates a semigroup $T_{t}$, then defining $x(t)=\left(T_{t} \phi\right)(0)$, we get a solution to (1.1). The standard procedure to show that an unbounded linear operator generates a semigroup, is to use the Hille-Yosida theorem $([8])$. But the estimations involved are difficult to obtain. It can be shown that for $\phi \in \mathbf{C}_{\sigma}(-\infty, 0],(1.1)$ has a unique solution and then we define the semigroup via the solution to (1.1). 
Lemma 2.3. Let $\phi \in \mathbf{C}_{\sigma}(-\infty, 0]$. The problem (1.1) has a unique solution $x$ : $\mathbb{R} \longrightarrow \mathbb{R}$. Further, for any $t \in[0, \infty)$, there is a constant $c(t)>0$ such that

$$
\sup _{s \in[0, t]}|x(s)| \leq c(t)\|\phi\|_{\sigma} .
$$

Sketch of the Proof. Consider $t \in\left[0, \tau_{1}\right] . \sum_{k=1}^{\infty} b_{k} \phi\left(t-\tau_{k}\right)$ converges uniformly on $\left[0, \tau_{1}\right]$. Further,

$$
\sup _{t \in\left[0, \tau_{1}\right]}\left|\sum_{k=1}^{\infty} b_{k} \phi\left(t-\tau_{k}\right)\right| \leq\|\phi\|_{\sigma}
$$

Define $y_{1}:\left[0, \tau_{1}\right] \rightarrow \mathbb{R}$ as the unique solution to the initial value problem

$$
\begin{aligned}
& x^{\prime}(t)=a x(t)+\sum_{k=1}^{\infty} b_{k} \phi\left(t-\tau_{k}\right), \\
& x(0)=\phi(0) .
\end{aligned}
$$

We have

$$
y_{1}(t)=\phi(0) e^{a t}+e^{a t} \int_{0}^{t} e^{-a s}\left(\sum_{k=1}^{\infty} b_{k} \phi\left(s-\tau_{k}\right)\right) d s .
$$

Define $x_{1}:\left(-\infty, \tau_{1}\right] \longrightarrow \mathbb{R}$ as

$$
\begin{aligned}
x_{1}(s) & =\phi(s), s \in(-\infty, 0] \\
& =y_{1}(s), s \in\left[0, \tau_{1}\right] .
\end{aligned}
$$

Clearly,

$$
\sup \left\{\left|x_{1}(t)\right|: t \in\left[0, \tau_{1}\right]\right\} \leq\left(\sup _{t \in\left[0, \tau_{1}\right]} e^{a t}\right)|\phi(0)|+\left(\sup _{t \in\left[0, \tau_{1}\right]} \frac{e^{a t}-1}{a}\right)\|\phi\|_{\sigma} .
$$

Here, note that for $r>0, \frac{e^{a r}-1}{a}>0$ for all $a \neq 0$. From the estimate

$$
\left|b_{1}\right|(|\phi(0)|) \leq\left|b_{1}\right| \sup _{\theta \in\left[-\tau_{1}, 0\right]}|\phi(\theta)| \leq\|\phi\|_{\sigma},
$$

we get

(2.4) $\sup \left\{\left|x_{1}(t)\right|: t \in\left[0, \tau_{1}\right]\right\} \leq\left(\sup _{t \in\left[0, \tau_{1}\right]} e^{a t}\right) \frac{1}{\left|b_{1}\right|}\|\phi\|_{\sigma}+\left(\sup _{t \in\left[0, \tau_{1}\right]} \frac{e^{a t}-1}{a}\right)\|\phi\|_{\sigma}$.

Now, we claim that for each $k \in \mathbb{N}$, there exists a function $x_{k}:\left(-\infty, k \tau_{1}\right]$ with the following properties:

(i) For each $t \in\left[0, k \tau_{1}\right]$,

$$
\sum_{i=1}^{\infty} b_{i} x_{k}\left(t-\tau_{i}\right)
$$

converges and this summation defines a continuous function on $\left[0, k \tau_{1}\right]$.

(ii) $x_{k}$ is the unique solution to

$$
\begin{aligned}
& x^{\prime}(t)=a x(t)+\sum_{k=1}^{\infty} b_{i} x_{k}\left(t-\tau_{i}\right), \text { for } t \in\left[0, k \tau_{1}\right], \\
& x(\theta)=\phi(\theta), \quad \text { for } t \in(-\infty, 0] .
\end{aligned}
$$

(iii) There exist constants $c_{k} \geq 0$ such that

$$
\sup \left\{\left|x_{k}(t)\right|: t \in\left[(k-1) \tau_{1}, k \tau_{1}\right]\right\} \leq c_{k}\left(\|\phi\|_{\sigma}\right) .
$$


The case $k=1$ is already proved and the above claim can be proved by induction.

The solution to (1.1) is obtained by patching up the functions $x_{k}$. Note that for every $t \geq 0$, there is $k$ such that $t \in\left[k \tau_{1},(k+1) \tau_{1}\right]$ With this $k$, define $c(t)=$ $\max \left\{c_{1}, c_{2}, \ldots, c_{k}\right\}$ and $(2.1)$ is proved.

Proof of Theorem 2.2. Let $\phi \in \mathbf{C}_{\sigma}(-\infty, 0]$ and $x_{\phi}$ be the unique solution to (1.1). Define $T_{t}(\phi)$ as

$$
\begin{aligned}
{\left[T_{t} \phi\right](\theta) } & =\phi(t+\theta), \quad \text { if } t+\theta \leq 0 \\
& =x_{\phi}(t+\theta) \text { if } t+\theta>0 .
\end{aligned}
$$

Fix $t \geq 0$. For $\theta \in\left[m_{k}, 0\right], t+\theta \leq\left[m_{k}, t\right]$. We can get the estimate

$$
\left\|T_{t} \phi\right\|_{\sigma}=\left(c(t)\|\mathbf{b}\|_{1}+1\right)\|\phi\|_{\sigma} .
$$

From the above estimate, it follows that $T_{t} \phi \in \mathbf{C}_{\sigma}(-\infty, 0]$ and that $T_{t}$ is a bounded linear map.

Next, we prove that

$$
\lim _{t \rightarrow 0}\left(T_{t} \phi-\phi\right)=0 .
$$

Define $p_{k}(\phi)=\sup _{\theta \in\left[m_{k}, 0\right]}|\phi(\theta)|$. Fix $k \in \mathbb{N}$ and consider

$$
\begin{aligned}
p_{k}\left(T_{t} \phi-\phi\right) & =\sup _{\theta \in\left[m_{k}, 0\right]}\left|\left(T_{t} \phi\right)(\theta)-\phi(\theta)\right| \\
& =\sup _{\theta \in\left[m_{k}, 0\right]}\left|x_{\phi}(t+\theta)-x_{\phi}(\theta)\right| .
\end{aligned}
$$

Clearly, $x$ is uniformly continuous in $\left[m_{k}, 1\right]$. Thus, given $\epsilon$, there is $\delta^{*}>0$ such that $\left|x\left(s_{1}\right)-x\left(s_{2}\right)\right| \leq \epsilon$ whenever $s_{1}, s_{2} \in\left[m_{k}, 1\right]$ and $\left|s_{1}-s_{2}\right|<\delta^{*}$. Now, let $\theta \in$ $\left[m_{k}, 0\right]$ and $|t| \leq \min \left(1, \delta^{*}\right)$. We have $m_{k} \leq t+\theta \leq 1$. Thus, $\left|x_{\phi}(t+\theta)-x_{\phi}(\theta)\right| \leq \epsilon$.

Thus, by taking $\delta=\min \left(1, \delta^{*}\right)$, the following holds: Given $\epsilon>0$, there is $\delta$ such that for all $|t| \leq \delta \Rightarrow p_{k}\left(T_{t} \phi-\phi\right) \leq \epsilon$. That is, for a fixed $k$,

$$
\lim _{t \rightarrow 0} p_{k}\left(T_{t} \phi-\phi\right)=0 \text {. }
$$

Now, for any $t \in[0,1]$,

$$
p_{k}\left(T_{t} \phi-\phi\right) \leq \max \left(p_{k}(\phi), \sup _{s \in[0,1]}|x(s)|\right) .
$$

Now, (2.7) follows from (2.8), (2.9), and Proposition 1.9.

It is easy to see that $T_{t+s}=T_{t} T_{s}$ and hence $T_{t}$ is a semi-group on $\mathbf{C}_{\sigma}(-\infty, 0]$. Further, we can show that $\lim _{h \rightarrow 0^{+}} \frac{T_{h} \phi-\phi}{h}$ exists if and only if $\phi \in D(A)$ and for such a $\phi$ this limit is equal to $\phi^{\prime}$. The proof is complete.

\section{The space $\mathbf{C}_{\sigma}(-\infty, 0]$ And a PieCEWise linear interpolation}

In this section, we construct a sequence of piecewise linear approximation $S_{n} \phi$ for elements of $\mathbf{C}_{\sigma}(-\infty, 0]$.

Let $m_{k}$ be as in Section 2 .

For a given $n \in \mathbb{N}$, consider the interval $\left[m_{n}, 0\right]$ and its $n$ sub-intervals $\left[m_{k}, m_{k-1}\right]$, $k=1,2, \ldots, n$. Divide each of these sub-intervals into $n$ intervals of length $=h_{k, n}=$ 
$\frac{m_{k-1}-m_{k}}{n}$. Thus, we get $n^{2}+1$ points $\theta_{i}, i=0,1,2, \ldots, n^{2}$ of the interval $\left[m_{n}, 0\right]$ which are given by

$$
\theta_{k n+j}=m_{k}+j\left(\frac{m_{k-1}-m_{k}}{n}\right),
$$

where $k=1,2, \ldots, n, j=0,1, \ldots,(n-1)$ and $m_{0}=0$.

The quantity $\omega(\phi, k, h)$, known as the modulus of continuity, is defined as

$$
\omega(\phi, k, h)=\sup \left\{\phi\left(\theta_{1}\right)-\phi\left(\theta_{2}\right)\left|: \theta_{1}, \theta_{2} \in\left[m_{k}, 0\right],\right| \theta_{1}-\theta_{2} \mid \leq h\right\} .
$$

The following result is well known:

For fixed $k \in \mathbb{N}$ and $\phi$ continuous on $\left[m_{k}, 0\right], \lim _{h \rightarrow 0} \omega(\phi, k, h)=0$.

Define $S_{n} \phi:(-\infty, 0] \longrightarrow \mathbb{R}$ as follows: On $\left[m_{n}, 0\right], S_{n} \phi$ is the piecewise linear interpolation to $\phi$ at the above $n^{2}+1$ points and on $\left(-\infty, m_{n}\right]$ it is identically equal to $\phi\left(m_{n}\right)$. Explicitly, for $\theta \in\left[\theta_{k n+j+1}, \theta_{k n+j}\right]$,

$$
S_{n} \phi(\theta)=\phi\left(\theta_{k n+j}\right)\left[1+\frac{\theta-\theta_{k n+j}}{h_{k, n}}\right]-\phi\left(\theta_{k n+j+1}\right)\left[\frac{\theta-\theta_{k n+j}}{h_{k, n}}\right] .
$$

Theorem 3.1. $S_{n} \phi$ converges to $\phi$ in the Banach space $\mathbf{C}_{\sigma}(-\infty, 0]$; that is,

$$
\lim _{n \rightarrow \infty}\left\|S_{n} \phi-\phi\right\|_{\sigma}=0
$$

Proof. We can also express $S_{n} \phi$ as

$$
S_{n} \phi=\sum_{i=0}^{n^{2}} \phi\left(\theta_{i}\right) B_{i}
$$

where $B_{i}:(-\infty, 0] \rightarrow \mathbb{R}^{\prime}$ s are defined as follows:

$$
\begin{aligned}
B_{0}(\theta) & =\frac{-\theta}{\theta_{1}}+1, \quad \theta \in\left[\theta_{1}, 0\right] \\
& =0 \text { elsewhere, } \\
B_{i}(\theta) & =\frac{\theta-\theta_{i+1}}{\theta_{i}-\theta_{i+1}}, \quad \theta \in\left[\theta_{i+1}, \theta_{i}\right] \\
& =\frac{\theta-\theta_{i-1}}{\theta_{i}-\theta_{i-1}}, \quad \theta \in\left[\theta_{i}, \theta_{i-1}\right] \\
& =0 \text { elsewhere, } \\
B_{n}^{2} & =\frac{\theta-\theta_{n^{2}-1}}{\theta_{n}^{2}-\theta_{n^{2}-1}}, \quad \theta \in\left[\theta_{n^{2}}, \theta_{n^{2}-1}\right] \\
& =1 \quad \theta \in\left(-\infty, m_{n}\right] \\
& =0 \text { elsewhere. }
\end{aligned}
$$

Since each $B_{i}$ is bounded and uniformly continuous, $B_{i} \in \mathbf{C}_{\sigma}(-\infty, 0]$ and hence $S_{n} \phi \in \mathbf{C}_{\sigma}(-\infty, 0]$. Let $h_{n, k}^{*}=\max \left\{h_{n, j}: j=1,2, \ldots, k\right\}$. Let $k$ be fixed and $n>k$. Note that $\theta_{n}=m_{1}, \theta_{2 n}=m_{2}, \ldots$, and $\theta_{n^{2}}=m_{n}$. Thus, for a fixed $k$, $\lim _{n \rightarrow \infty} h_{n, k}^{*}=0$. The estimate

$$
p_{k}\left(\phi-S_{n} \phi\right) \leq 3 \omega\left(\phi, m_{k}, h_{n, k}^{*}\right)
$$

is easily obtained. Now, since $k$ is fixed and $\lim _{n \rightarrow \infty} h_{n, k}^{*}=0$, we have

$$
\lim _{n \rightarrow \infty} p_{k}\left(S_{n} \phi-\phi\right)=0 \text {. }
$$


For all $k$, we have $p_{k}\left(S_{n} \phi\right) \leq p_{k}(\phi)$. Finally, for all $k$, we obtain

$$
p_{k}\left(S_{n} \phi-\phi\right) \leq 2 p_{k}(\phi)
$$

Now the convergence of $S_{n} \phi$ to $\phi$ follows from (3.1), (3.2), and Proposition 1.9.

\section{A Finite DifferenCE SCHEME FOR THE INFINIte DELAY EQUATION}

In this section we show the convergence of a finite difference scheme to the solution of the infinite delay equation.

Let $\theta_{i}, i=0,1,2, \ldots, n^{2}$ be as in the previous section. Define $P_{n}: \mathbf{C}_{\sigma}(-\infty, 0] \rightarrow$ $\mathbb{R}^{n^{2}+1}$ as $\left[P_{n}(\phi)\right]_{i}=\phi\left(\theta_{i}\right)$. Further, define $E_{n}: \mathbb{R}^{n^{2}+1} \longrightarrow \mathbf{C}_{\sigma}(-\infty, 0]$ as follows:

For $v \in \mathbb{R}^{n^{2}+1}, E_{n} v$ is the piecewise linear function taking values $\left(E_{n} v\right)\left(\theta_{i}\right)=v_{i}$ and $\left(E_{n} v\right)(\theta)=v_{n^{2}}$ for all $\theta \in\left(-\infty, m_{n}\right]$. Clearly, $E_{n} v=\sum_{i=0}^{n^{2}} v_{i} B_{i}$.

Now, consider $\left\|E_{n} v\right\|_{\sigma}$. From the definitions of $E_{n} v$ and the norm $\|\cdot\|_{\sigma}$, it is clear that

$$
\left\|E_{n} v\right\|_{\sigma}=\sum_{k=1}^{n}\left|b_{k}\right| \max \left\{\left|v_{0}\right|,\left|v_{1}\right|, \ldots,\left|v_{k n}\right|\right\}+\sum_{k=n+1}^{\infty}\left|b_{k}\right| \max \left\{\left|v_{0}\right|,\left|v_{1}\right|, \ldots,\left|v_{n^{2}}\right|\right\} .
$$

The following result holds:

\section{Proposition 4.1.}

(1) $\left\|P_{n}\right\| \leq M,\left\|E_{n}\right\|_{n} \leq 1$ where $M$ is independent of $n$,

(2) $\left\|E_{n} P_{n} \phi-\phi\right\|_{\sigma} \longrightarrow 0$ as $n \rightarrow \infty$ for all $\phi \in \mathbf{C}_{\sigma}$.

(3) $P_{n} E_{n}=I_{n}$ where $I_{n}$ is the identity operator on $X_{n}$.

Proof. We find that if \|\|$_{n}$ is the norm in $\mathbb{R}^{n^{2}+1}$ as defined in Section 1 , then $\left\|E_{n} v\right\|_{\sigma}=\|v\|_{n}$. Thus $\left\|E_{n}\right\|=1$. Now, $E_{n} P_{n} \phi$ is nothing but $S_{n} \phi$ where $S_{n}$ is the piecewise linear approximation of $\phi$ defined in Section 3. So, $\lim _{n \rightarrow \infty} E_{n} P_{n} \phi=$ $\lim _{n \rightarrow \infty} S_{n} \phi=\phi$ and hence (2) holds. By the uniform boundedness principle, we have the existence of $M \geq 0$ such that $\left\|S_{n}\right\| \leq M$ for all $n \in \mathbb{N}$. Now $\left\|P_{n} \phi\right\|_{n}=$ $\left\|E_{n} P_{n} \phi\right\|_{\sigma}=\left\|S_{n} \phi\right\|_{\sigma} \leq M\|\phi\|_{\sigma}$. Thus, (1) holds. (3) is obvious from the definition of $P_{n}$ and $E_{n}$.

Next, we discretise $A$ by using finite differences as follows: Define $A_{n}: \mathbb{R}^{n^{2}+1} \rightarrow$ $\mathbb{R}^{n^{2}+1}$ as

$$
\begin{aligned}
\left(A_{n}(v)\right)_{i} & =\frac{\left(v_{i-1}-v_{i}\right)}{\theta_{i-1}-\theta_{i}}, i=1,2,3, \ldots, n^{2}, \\
\left(A_{n}(v)\right)_{0} & =L\left[\left(E_{n} v\right)\right] .
\end{aligned}
$$

Theorem 4.2. Let $A$ and $T_{t}$ be as in Proposition 2.1 and let $A_{n}$ be as above. Let $T_{t}^{(n)}$ be the semigroup generated by $A_{n}$. We have

$$
\lim _{n \rightarrow \infty}\left\|E_{n} T_{t}^{(n)} P_{n} \phi-T_{t}(\phi)\right\|_{\sigma}=0
$$

We shall apply Theorem 1.7 to prove this result. First, we need the following lemma.

Lemma 4.3. Fix $v \in \mathbb{R}^{n^{2}+1}$. Define the function $l:\{1,2, \ldots, n\} \rightarrow\left\{0,1,2, \ldots, n^{2}\right\}$ as

$$
l(k)=\max \left\{0 \leq i \leq k n:\left|v_{i}\right| \geq\left|v_{j}\right| \text { for all } j \in\{0,1, . . k n\}\right\} .
$$


Define $\xi \in\left(\mathbb{R}^{n^{2}+1}\right)^{*}$ as follows:

$$
\langle w, \xi\rangle=\sum_{k=1}^{n}\left|b_{k}\right| \operatorname{sign} v_{l(k)} w_{l(k)}+\sum_{k=n+1}^{\infty}\left|b_{k}\right| \operatorname{sign} v_{l(n)} w_{l(n)} .
$$

Then, the continuous linear functional $\eta$ is defined as $\eta=\|v\| \xi \in F(v)$.

Proof.

$$
\begin{aligned}
|\langle w, \xi\rangle| & \leq \sum_{k=1}^{n}\left|b_{k}\right|\left|w_{l(k)}\right|+\sum_{k=n+1}^{\infty}\left|b_{k}\right|\left|w_{l(n)}\right| \\
& \leq \sum_{k=1}^{n}\left|b_{k}\right| \max \left\{\left|w_{i}\right|: 0 \leq i \leq k n\right\}+\sum_{k=n+1}^{\infty}\left|b_{k}\right| \max \left\{\left|w_{i}\right|: 0 \leq i \leq n^{2}\right\} \\
& =\|w\|_{n} .
\end{aligned}
$$

Furthermore,

$$
\begin{aligned}
\langle v, \xi\rangle & =\sum_{k=1}^{n}\left|b_{k}\right|\left|v_{l(k)}\right|+\sum_{k=n+1}^{\infty}\left|b_{k}\right|\left|v_{l(n)}\right| \\
& =\sum_{k=1}^{n}\left|b_{k}\right| \max \left\{\left|v_{i}\right|: 0 \leq i \leq k n\right\}+\sum_{k=n+1}^{\infty}\left|b_{k}\right| \max \left\{\left|v_{i}\right|: 0 \leq i \leq n^{2}\right\} \\
& =\|v\|_{n} .
\end{aligned}
$$

So, it is clear that $\|\xi\|=1$. Now, it is easy to see that $\eta=\|v\| \xi \in F(v)$.

Proof of Theorem 4.2. Since $A_{n}$ is a bounded linear operator, it generates the semigroup $T_{t}^{(n)}=e^{t A_{n}}$. We prove that each $A_{n}$ is in the class $G\left(1, \omega, X_{n}\right)$ where $\omega>\|L\|\|\mathbf{b}\|$. Consider $B_{n}=A_{n}-\omega I_{n}$ where $\omega>\|L\|\|\mathbf{b}\|$ and $I_{n}$ is the identity operator on $\mathbb{R}^{n^{2}+1}$. We prove that there exists $\eta \in F(v)$ such that $\left\langle B_{n} v, \eta\right\rangle \leq 0$. For $v \in D\left(A_{n}\right)=\mathbb{R}^{n^{2}+1}$, let $\xi \in\left(\mathbb{R}^{n^{2}+1}\right)^{*}$ and $\eta \in F(v)$ be as in the lemma above. Let us observe that for all $k=1, \ldots, n$ with $l(k) \neq 0,\left|v_{l(k)}\right| \geq\left|v_{l(k)-1}\right|$. Thus,

$$
\begin{aligned}
\left|\sum_{k \in l^{-1}(0)}\right| b_{k}\left|\operatorname{sign}\left(v_{l(k)}\right) L\left(E_{n} v\right)\right|+\left|\sum_{k=n+1}^{\infty}\right| b_{k}\left|\operatorname{sign}\left(v_{l(n)}\right) L\left(E_{n} v\right)\right| & \leq\|\mathbf{b}\|\|L\|\left\|E_{n}\right\|\|v\|_{n} \\
& \leq(\|\mathbf{b}\|\|L\|)\|v\|_{n} .
\end{aligned}
$$

Now,

$$
\begin{aligned}
\left\langle B_{n} v, \xi\right\rangle & =\left\langle A_{n} v, \xi\right\rangle-\langle\omega v, \xi\rangle \\
& =\sum_{k=1}^{n}\left|b_{k}\right| \operatorname{sign}\left(v_{l(k)}\right)\left(A_{n} v\right)_{l(k)}+\sum_{k=n+1}^{\infty}\left|b_{k}\right| \operatorname{sign}\left(v_{l(n)}\right)\left(A_{n} v\right)_{l(n)}-\omega\|v\|_{n} \\
& =\sum_{k \notin l^{-1}(0)}\left|b_{k}\right| \operatorname{sign}\left(v_{l(k)}\right) \frac{v_{l(k)-1}-v_{l(k}}{\theta_{l(k)-1}-\theta_{l(k)}}+\sum_{k \in l^{-1}(0)}\left|b_{k}\right| \operatorname{sign}\left(v_{l(k)}\right) L\left(E_{n} v\right) \\
& +\sum_{k=n+1}^{\infty}\left|b_{k}\right| \operatorname{sign}\left(v_{l(n)}\right)\left(A_{n} v\right)_{l(n)}-\omega\|v\|_{n} .
\end{aligned}
$$


Now we consider two cases, namely $l(n)=0$ and $l(n) \neq 0$. If $l(n)=0$, then $\left(A_{n} v\right)_{l(n)}=L\left(E_{n} v\right)$ and we have the estimate

$$
\begin{aligned}
\left\langle B_{n} v, \xi\right\rangle & =\sum_{k \notin l^{-1}(0)}\left|b_{k}\right| \operatorname{sign}\left(v_{l(k)}\right) \frac{v_{l(k)-1}-v_{l(k}}{\theta_{l(k)-1}-\theta_{l(k)}} \\
& +\sum_{k \in l^{-1}(0)}\left|b_{k}\right| \operatorname{sign}\left(v_{l(k)}\right) L\left(E_{n} v\right) \\
& +\sum_{k=n+1}^{\infty}\left|b_{k}\right| \operatorname{sign}\left(v_{l(n)}\right) L\left(E_{n} v\right)-\omega\|v\|_{n} \\
\leq & \sum_{k \in l^{-1}(0)}\left|b_{k}\right| \operatorname{sign}\left(v_{l(k)}\right) L\left(E_{n} v\right) \\
& +\sum_{k=n+1}^{\infty}\left|b_{k}\right| \operatorname{sign}\left(v_{l(n)}\right) L\left(E_{n} v\right)-\omega\|v\|_{n} \\
\leq & 0 .
\end{aligned}
$$

If $l(n) \neq 0$, then $\left(A_{n} v\right)_{l(n)}=\frac{v_{l(n)-1}-v_{l(n)}}{\theta_{l(n)-1}-\theta_{l(n)}}$ and we have the estimate

$$
\begin{aligned}
\left\langle B_{n} v, \xi\right\rangle= & \sum_{k \notin l^{-1}(0)}\left|b_{k}\right| \operatorname{sign}\left(v_{l(k)}\right) \frac{v_{l(k)-1}-v_{l(k}}{\theta_{l(k)-1}-\theta_{l(k)}} \\
& +\sum_{k \in l^{-1}(0)}\left|b_{k}\right| \operatorname{sign}\left(v_{l(k)}\right) L\left(E_{n} v\right) \\
& +\sum_{k=n+1}^{\infty}\left|b_{k}\right| \operatorname{sign}\left(v_{l(n)}\right) \frac{v_{l(n)-1}-v_{l(n)}}{\theta_{l(n)-1}-\theta_{l(n)}}-\omega\|v\|_{n} \\
\leq & \sum_{k \in l^{-1}(0)}\left|b_{k}\right| \operatorname{sign}\left(v_{l(k)}\right) L\left(E_{n} v\right)-\omega\|v\|_{n} \\
\leq & 0 .
\end{aligned}
$$

Thus, $\left\langle B_{n} v, \eta\right\rangle=\left\langle B_{n} v,\|v\|_{n} \xi\right\rangle \leq 0$. It is elementary to check that if $\lambda$ is a real eigenvalue of $B_{n}$, then $\lambda \leq 0$. Thus, since $\mathbb{R}^{n^{2}+1}$ is finite dimensional, if $\lambda>0$, then $\lambda I_{n}-B_{n}$ is invertible and in particular onto. Thus, by Theorem 1.6, it follows that $B_{n}=A_{n} v-\lambda I_{n}$ generates a contraction semigroup. But $e^{-\omega t} T_{t}^{n}$ is the semigroup generated by $B_{n}$. Thus, $\left\|e^{-\omega t} T_{t}^{n}\right\| \leq 1$ for all $n$. We get $\left\|T_{t}^{n}\right\| \leq e^{\omega t}$. Let $T_{t}$ be of class $G\left(M_{A}, \omega_{A}, X\right)$. Now, let $\omega_{0}=\max \left\{\omega_{A}, \omega\right\}$. We have now proved that $A \in G\left(M_{A}, \omega_{0}, X\right)$ and $A_{n} \in G\left(M_{A}, \omega_{0}, X_{n}\right)$.

To complete the proof, we need to check (a) of Theorem 1.7. Choose $\phi \in D(A)$ and $v_{n} \in \mathbb{R}^{n^{2}+1}$ as $v_{n}=P_{n}(\phi)$. Thus, $\left(v_{n}\right)_{i}=\phi\left(\theta_{i}\right)$ and $E_{n} v_{n}=E_{n} P_{n} \phi=S_{n} \phi$. So, $\lim _{n \rightarrow \infty} E_{n} v_{n}=\phi$. Next, we have to prove that $E_{n} A_{n} v_{n} \rightarrow A \phi$.

By the mean value theorem, for every $i=1,2, \ldots, n^{2}$ there exists $\zeta_{i} \in\left[\theta_{i}, \theta_{i-1}\right]$ such that

$$
\phi^{\prime}\left(\zeta_{i}\right)=\frac{\phi\left(\theta_{i-1}\right)-\phi\left(\theta_{i}\right)}{\theta_{i-1}-\theta_{i}}
$$


Therefore,

$$
\begin{aligned}
& \left.\| E_{n} A_{n} v_{n}(x)-A \phi\right) \|_{\sigma} \\
& \quad=\left\|\sum_{i=1}^{n^{2}} \frac{\left(\phi\left(\theta_{i-1}\right)-\phi\left(\theta_{i}\right)\right.}{\theta_{i-1}-\theta_{i}} B_{i}+L\left(S_{n} \phi\right) B_{0}-\phi^{\prime}\right\|_{\sigma} \\
& \quad=\left\|\sum_{1=1}^{n^{2}} \phi^{\prime}\left(\zeta_{i}\right) B_{i}+L\left(S_{n} \phi\right) B_{0}-\phi^{\prime}\right\|_{\sigma} \\
& \quad \leq\left\|\sum_{i=1}^{n^{2}} \phi^{\prime}\left(\zeta_{i}\right) B_{i}+L\left(S_{n} \phi\right) B_{0}-\sum_{i=0}^{n^{2}} \phi^{\prime}\left(x_{i}\right) B_{i}\right\|_{\sigma}+\left\|\sum_{i=0}^{n^{2}} \phi^{\prime}\left(\theta_{i}\right) B_{i}-\phi^{\prime}\right\|_{\sigma} \\
& \quad \leq\left\|\sum_{i=1}^{n^{2}}\left(\phi^{\prime}\left(\zeta_{i}\right)-\phi^{\prime}\left(\theta_{i}\right)\right) B_{i}+L\left(S_{n} \phi\right) B_{0}-\phi^{\prime}(0) B_{0}\right\|_{\sigma}+\left\|\sum_{i=0}^{n^{2}} \phi^{\prime}\left(\theta_{i}\right) B_{i}-\phi^{\prime}\right\|_{\sigma} .
\end{aligned}
$$

Now, $\left\|\sum_{k=0}^{n^{2}} \phi^{\prime}\left(x_{i}\right) B_{i}-\phi^{\prime}\right\|_{\sigma}=\left\|S_{n}\left(\phi^{\prime}\right)-\phi^{\prime}\right\|_{\sigma}$ and hence by Theorem 3.1, $\lim _{n \rightarrow \infty}\left\|\sum_{k=0}^{n^{2}} \phi^{\prime}\left(\theta_{i}\right) B_{i}-\phi^{\prime}\right\|_{\sigma}=0$. We need to prove that

$$
\lim _{n \rightarrow \infty}\left\|\sum_{i=1}^{n^{2}}\left(\phi^{\prime}\left(\zeta_{i}\right)-\phi^{\prime}\left(\theta_{i}\right)\right) B_{i}+L\left(S_{n} \phi\right) B_{0}-\phi^{\prime}(0) B_{0}\right\|_{\sigma}=0 .
$$

Since $\lim _{n \rightarrow \infty} L\left(S_{n} \phi\right)=L(\phi)$, there is $c>0$ such that $\left|L\left(S_{n} \phi\right)\right| \leq c$ for all $n$. For $k<n$,

$$
p_{k}\left(\sum_{i=1}^{n^{2}}\left(\phi^{\prime}\left(\zeta_{i}\right)-\phi^{\prime}\left(\theta_{i}\right)\right) B_{i}+L\left(S_{n} \phi\right) B_{0}-\phi^{\prime}(0) B_{0}\right) \leq 3 p_{k}\left(\phi^{\prime}\right)+c .
$$

For $k \geq n$,

$$
p_{k}\left(\sum_{i=1}^{n^{2}}\left(\phi^{\prime}\left(\zeta_{i}\right)-\phi^{\prime}\left(\theta_{i}\right)\right) B_{i}+L\left(S_{n} \phi\right) B_{0}-\phi^{\prime}(0) B_{0}\right) \leq 3 p_{n}\left(\phi^{\prime}\right)+c \leq 3 p_{k}\left(\phi^{\prime}\right)+c .
$$

So, we have proved that for all $k$,

$$
p_{k}\left(\sum_{i=1}^{n^{2}}\left(\phi^{\prime}\left(\zeta_{i}\right)-\phi^{\prime}\left(\theta_{i}\right)\right) B_{i}+L\left(S_{n} \phi\right) B_{0}-\phi^{\prime}(0) B_{0}\right) \leq 3 p_{k}\left(\phi^{\prime}\right)+c .
$$

Now, fix $k<n . B_{i} \equiv 0$ on $\left[m_{k}, 0\right]$ for $i>k n$. Thus,

$$
\begin{aligned}
p_{k}\left(\sum_{i=1}^{n^{2}} \phi^{\prime}\left(\zeta_{i}\right) B_{i}-\sum_{i=1}^{n^{2}} \phi^{\prime}\left(x_{i}\right) B_{i}\right) & =\sup _{x \in\left[m_{k}, 0\right]}\left|\sum_{i=1}^{n^{2}} \phi^{\prime}\left(\zeta_{i}\right) B_{i}-\sum_{i=1}^{n^{2}} \phi^{\prime}\left(x_{i}\right) B_{i}\right| \\
& =\sup _{x \in\left[m_{k}, 0\right]}\left|\sum_{i=1}^{k n}\left(\phi^{\prime}\left(\zeta_{i}\right)-\phi^{\prime}\left(x_{i}\right)\right) B_{i}\right| \\
& \leq \omega\left(\phi^{\prime}, k, h_{n, k}^{*}\right) .
\end{aligned}
$$

Thus, $\lim _{n \rightarrow \infty} p_{k}\left(\sum_{i=1}^{n^{2}} \phi^{\prime}\left(\zeta_{i}\right) B_{i}-\sum_{k=1}^{n^{2}} \phi^{\prime}\left(x_{i}\right) B_{i}\right)=0$. Now,

$$
p_{k}\left[\left(L\left(S_{n} \phi\right)-\phi^{\prime}(0)\right) B_{0}\right] \leq\left|L\left(S_{n} \phi\right)-\phi^{\prime}(0)\right| .
$$


Hence $\lim _{n \rightarrow \infty} p_{k}\left(\left(L\left(S_{n} \phi\right)-\phi^{\prime}(0)\right) B_{0}\right)=0$ Finally, we have

$$
\lim _{n \rightarrow \infty} p_{k}\left(\sum_{i=1}^{n^{2}} \phi^{\prime}\left(\zeta_{i}\right) B_{i}-\sum_{k=1}^{n^{2}} \phi^{\prime}\left(x_{i}\right) B_{i}+\left(L\left(S_{n}(\phi)\right)-\phi^{\prime}(0)\right) B_{0}\right)=0 .
$$

From (4.2), (4.3) and Proposition 1.9, (4.1) follows.

\section{NUMERICAL EXAMPLES}

In this section we give examples where the initial functions $\phi$ is neither bounded nor integrable

\section{Example 1.}

$$
\begin{aligned}
& x^{\prime}(t)=x(t)+x(t-0.5)+\sum_{k=2}^{\infty} \frac{1}{k^{2}} x(t-k), t>0, \\
& x(\theta)=\sqrt{-\theta}, \quad-\infty<\theta \leq 0 .
\end{aligned}
$$

In this example, $\tau_{1}=0.5$ and for $k \geq 2, \tau_{k}=k$. For $k \geq 1, b_{k}=\frac{1}{k^{2}}, m_{k}=-k$.

Thus, $\theta_{i}=-\frac{i}{n}, i=0,1,2, \ldots, n^{2}$. Let $n$ be even. So, $-0.5=-\tau_{1}=\theta_{n / 2}$ and for $k \geq 2, \tau_{k}=k=-\theta_{k n}$. Since

$$
L(\phi)=a \phi(0)+\phi(-0.5)+\sum_{k=2}^{\infty} \phi(-k)
$$

we obtain that

$$
L\left(E_{n} v\right)=v_{0}+v_{n / 2}+\sum_{k=2}^{n} \frac{1}{k^{2}} v_{k n}+\left(\sum_{k=n+1}^{\infty} \frac{1}{k^{2}}\right) v_{k n} .
$$

With reference to Theorem $4.2,\left(T_{t}^{(n)}\right) v$ is the solution to the system

$$
\begin{aligned}
v_{0}^{\prime}(t) & =v_{0}(t)+v_{n / 2}(t)+\sum_{k=2}^{n} \frac{1}{k^{2}} v_{k n}(t)+\left(\sum_{k=n+1}^{\infty} \frac{1}{k^{2}}\right) v_{k n}(t), \\
v_{i}^{\prime}(t) & =n\left(v_{i-1}(t)-v_{i}(t)\right), \quad i=1,2, \ldots, n^{2} \\
v(0) & =v .
\end{aligned}
$$

Taking $v=P_{n} \phi$ in (5.2), $\left(E_{n} T_{t}^{(n)} P_{n} \phi\right)\left(\theta_{i}\right)$ is nothing but $v_{i}(t)$.

Now, let $x$ be the unique solution to (5.1). Then $\left(T_{t} \phi\right)\left(\theta_{i}\right)=x\left(t+\theta_{i}\right)$.

As per Theorem $4.2, v_{i}(t)$ is an approximation to $x\left(t+\theta_{i}\right)$.

$x$ satisfies the equation

$$
\begin{aligned}
& x^{\prime}(t)=x(t)+\sqrt{0.5-t}+\sum_{k=2}^{\infty} \frac{1}{k^{2}} \sqrt{k-t}, \\
& x(0)=0,
\end{aligned}
$$

for $t \in[0,0.5]$. It is clear that the above equation has no closed form solution. Using the Matlab ODE45 function, we have calculated the values of the solution at $t=0,0.1,0.2,0.3,0.4,0.5$ to the equation

$$
\begin{aligned}
& x^{\prime}(t)=x(t)+\sqrt{0.5-t}+\sum_{k=2}^{1,00,000} \frac{1}{k^{2}} \sqrt{k-t} \\
& x(0)=0 .
\end{aligned}
$$


The above solution is denoted by $x^{*}$.

We solve the system (5.2) using the Matlab ODE45 function. In the table below, we take $i=0$ and compare the values of $v_{0}(t)$ and $x^{*}(t)$ which is an approximation of $x(t)$ in the interval $[0,0.5]$. We consider $t=0,0.1,0.2,0.3,0.4,0.5$ and $n=$ $10,20,60,70$.

\begin{tabular}{|c|c|c|c|c|c|}
\hline$t$ & $\begin{array}{c}n=10 \\
v_{0}(t)\end{array}$ & $\begin{array}{c}n=20 \\
v_{0}(t)\end{array}$ & $\begin{array}{c}n=60 \\
v_{0}(t)\end{array}$ & $\begin{array}{c}n=70 \\
v_{0}(t)\end{array}$ & $x^{*}(t)$ \\
\hline 0 & 0 & 0 & 0 & 0 & 0 \\
0.1 & 0.2090 & 0.2168 & 0.2256 & 0.2268 & 0.2386 \\
0.2 & 0.4288 & 0.4457 & 0.4647 & 0.4671 & 0.4921 \\
0.3 & 0.6593 & 0.6862 & 0.7170 & 0.7209 & 0.7608 \\
0.4 & 0.9036 & 0.9386 & 0.9810 & 0.9866 & 1.0439 \\
0.5 & 1.1685 & 1.2083 & 1.2573 & 1.2640 & 1.3364 \\
0.6 & 1.4631 & 1.5062 & 1.5589 & 1.5664 & - \\
0.7 & 1.7972 & 1.8449 & 1.9051 & 1.9141 & - \\
0.8 & 2.1807 & 2.2359 & 2.3094 & 2.3206 & - \\
0.9 & 2.6236 & 2.6896 & 2.7810 & 2.7948 & - \\
1.0 & 3.1364 & 3.2161 & 3.3285 & 3.3454 & - \\
\hline
\end{tabular}

Remark. To approximately evaluate $x$ in $[0.5,2]$, we may use a linear interpolation of $x$ in $[0,0.5]$. Then, to approximately evaluate $x$ in $[2,3]$, we may use a linear interpolation of $x$ in $[0.5,2]$ and so on. But the advantage in our procedure is that we use only the initial data $\phi$ to evaluate $x(t)$ for any $t$.

Let $n=70$. For a fixed $t, T_{t}(\phi) \in C_{\sigma}(-\infty, 0]$. Now, $E_{n} T_{t}^{n} P_{n}(\phi)\left(\theta_{i}\right)=v_{i}(t)$. For each $t$, an approximation to $T_{t}(\phi)$ is obtained by interpolating the 4901 values $v_{i}(t)$ for $i=0,1, \ldots, 4901$. In the following tables, we tabulate values of $v_{i}(t)$ for some values of $i$. Whenever $t+\theta_{i} \leq 0$, we compare these values with $T_{t} \phi\left(\theta_{i}\right)=$ $x\left(t+\theta_{i}\right)=\sqrt{\left|t+\theta_{i}\right|}:$

\begin{tabular}{|c|c|c|c|c|c|c|}
\hline & \multicolumn{2}{|c|}{$\theta=0$} & \multicolumn{2}{|c|}{$\theta=-1 / 70$} & \multicolumn{2}{|c|}{$\theta=-1 / 7$} \\
\hline & Num. & Act. & Num & Act. & Num. & Act \\
\hline $\mathrm{t}=0$ & 0 & 0 & 0.1195 & 0.1195 & 0.3780 & 0.3780 \\
\hline $\mathrm{t}=0.1$ & 0.2268 & - & 0.1938 & - & 0.1945 & 0.2070 \\
\hline $\mathrm{t}=0.2$ & 0.4671 & - & 0.4321 & - & 0.1543 & - \\
\hline $\mathrm{t}=0.3$ & 0.7209 & - & 0.6839 & - & 0.3644 & - \\
\hline $\mathrm{t}=0.4$ & 0.9866 & - & 0.9481 & - & 0.6118 & - \\
\hline $\mathrm{t}=0.5$ & 1.2640 & - & 1.2236 & - & 0.8725 & - \\
\hline $\mathrm{t}=0.6$ & 1.5664 & - & 1.5212 & - & 1.1448 & - \\
\hline $\mathrm{t}=0.7$ & 1.9141 & - & 1.8616 & - & 1.4359 & - \\
\hline $\mathrm{t}=0.8$ & 2.3206 & - & 2.2592 & - & 1.7638 & - \\
\hline $\mathrm{t}=0.9$ & 2.7948 & - & 2.7234 & - & 2.1450 & - \\
\hline $\mathrm{t}=1$ & 3.3454 & - & 3.2626 & - & 2.5901 & - \\
\hline
\end{tabular}




\begin{tabular}{|c|c|c|c|c|c|c|}
\hline & \multicolumn{2}{|c|}{$\theta=-4 / 7$} & \multicolumn{2}{|c|}{$\theta=-1$} & \multicolumn{2}{|c|}{$\theta=-8 / 7$} \\
\hline & Num & Act. & Num. & Act. & Num. & Act. \\
\hline$t=0$ & 0.7559 & 0.7559 & 1.0000 & 1.0000 & 1.0690 & 1.0690 \\
\hline $\mathrm{t}=0.1$ & 0.6860 & 0.6866 & 0.9485 & 0.9487 & 1.0210 & 1.0212 \\
\hline $\mathrm{t}=0.2$ & 0.6078 & 0.6094 & 0.8939 & 0.8944 & 0.9706 & 0.9710 \\
\hline $\mathrm{t}=0.3$ & 0.5168 & 0.5300 & 0.8357 & 0.8367 & 0.9174 & 0.9181 \\
\hline $\mathrm{t}=0.4$ & 0.4016 & 0.4140 & 0.7730 & 0.7746 & 0.8608 & 0.8619 \\
\hline $\mathrm{t}=0.5$ & 0.2619 & 0.2672 & 0.7045 & 0.7071 & 0.8000 & 0.8018 \\
\hline $\mathrm{t}=0.6$ & 0.2073 & - & 0.6278 & 0.6325 & 0.7340 & 0.7368 \\
\hline $\mathrm{t}=0.7$ & 0.3258 & - & 0.5388 & 0.5477 & 0.6610 & 0.6655 \\
\hline $\mathrm{t}=0.8$ & 0.5465 & - & 0.4297 & 0.4472 & 0.5776 & 0.5855 \\
\hline $\mathrm{t}=0.9$ & 0.8007 & - & 0.3118 & 0.3162 & 0.4771 & 0.4928 \\
\hline$t=1$ & 1.0704 & - & 0.2517 & 0.0000 & 0.3618 & 0.3780 \\
\hline
\end{tabular}

\begin{tabular}{|c|c|c|c|c|}
\hline & \multicolumn{2}{|c|}{$\theta=-20$} & \multicolumn{2}{|c|}{$\theta=-70$} \\
\hline & Num. & Act & Num & Act. \\
\hline $\mathrm{t}=0$ & 4.4721 & 4.4721 & 8.3666 & 8.3666 \\
\hline $\mathrm{t}=0.1$ & 4.4609 & 4.4609 & 8.3606 & 8.3606 \\
\hline $\mathrm{t}=0.2$ & 4.4497 & 4.4497 & 8.3546 & 8.3546 \\
\hline $\mathrm{t}=0.3$ & 4.4385 & 4.4385 & 8.3487 & 8.3487 \\
\hline $\mathrm{t}=0.4$ & 4.4272 & 4.4272 & 8.3427 & 8.3427 \\
\hline $\mathrm{t}=0.5$ & 4.4159 & 4.4159 & 8.3367 & 8.3367 \\
\hline $\mathrm{t}=0.6$ & 4.4045 & 4.4045 & 8.3307 & 8.3307 \\
\hline $\mathrm{t}=0.7$ & 4.3932 & 4.3932 & 8.3247 & 8.3247 \\
\hline $\mathrm{t}=0.8$ & 4.3818 & 4.3818 & 8.3187 & 8.3126 \\
\hline $\mathrm{t}=0.9$ & 4.3703 & 4.3703 & 8.3126 & 8.3216 \\
\hline $\mathrm{t}=1$ & 4.3589 & 4.3589 & 8.3066 & 8.3066 \\
\hline
\end{tabular}

Example 2. Consider

$$
\begin{aligned}
x^{\prime}(t) & =x(t)+x(t-0.5)+\sum_{k=2}^{\infty} \frac{1}{k^{3}} x(t-k), t>0, \\
x(\theta) & =\theta,-\infty<\theta \leq 0 .
\end{aligned}
$$

It is elementary to show that the solution is

$$
\begin{aligned}
x(t) & =-\alpha t+(\alpha-\beta)\left(e^{t}-1\right), 0 \leq t \leq 0.5 \\
& =-p t+(p-q)\left(e^{t-0.5}-1\right)+r(t-(0.5)) e^{t}+e^{t-0.5}, 0.5<t \leq 1
\end{aligned}
$$

where $\alpha=\sum_{i=1}^{\infty} \frac{1}{i^{3}}, \beta=-0.5-\sum_{i=2}^{\infty} \frac{1}{i^{2}} p=-1 q=-\sum_{i=2}^{\infty} \frac{1}{i^{2}}+0.5 \alpha$ and $r=(\alpha+\beta) e^{-0.5}$.

In this example,

$$
L\left(E_{n} \phi\right)=\phi(0)+\phi(-0.5)+\sum_{k=2}^{n} \frac{1}{k^{3}} \phi(-k)+\sum_{k=n+1}^{\infty} \frac{1}{k^{3}} \phi(-n) .
$$


We solve the system

$$
\begin{aligned}
v_{0}^{\prime}(t) & =v_{0}(t)+v_{n / 2}(t)+\sum_{k=2}^{n} \frac{1}{k^{3}} v_{k n}(t)+\left(\sum_{k=n+1}^{\infty} \frac{1}{k^{3}}\right) v_{k n}, \\
v_{i}^{\prime}(t) & =n\left(v_{i-1}-v_{i}\right), \quad i=1,2, \ldots, n^{2}, \\
v(0) & =v,
\end{aligned}
$$

with $v=P_{n} \phi$.

Unlike in Example 1, we know the exact solution for $t \in[0,1]$ and we compare $v_{0}(t)$ and $x(t)$ in the following table for various values of $n$ :

\begin{tabular}{|c|c|c|c|c|c|}
\hline$t$ & $n=10$ & $n=20$ & $n=50$ & $n=60$ & \\
& $v_{0}(t)$ & $v_{0}(t)$ & $v_{0}(t)$ & $v_{0}(t)$ & $x(t)$ \\
\hline 0 & 0 & 0 & 0 & 0 & 0 \\
0.1 & -0.1089 & -0.1116 & -0.1131 & -0.1133 & -0.1142 \\
0.2 & -0.2168 & -0.2223 & -0.2256 & -0.2259 & -0.2278 \\
0.3 & -0.3247 & -0.3323 & -0.3371 & -0.3377 & -0.3406 \\
0.4 & -0.4355 & -0.4433 & -0.4483 & -0.4490 & -0.4527 \\
0.5 & -0.5533 & -0.5597 & -0.5625 & -0.5628 & -0.5639 \\
0.6 & -0.6831 & -0.6876 & -0.6876 & -0.6874 & -0.6853 \\
0.7 & -0.8295 & -0.8329 & -0.8317 & -0.8313 & -0.8293 \\
0.8 & -0.9970 & -1.0005 & -0.9996 & -0.9994 & -0.9982 \\
0.9 & -1.1899 & -1.1946 & -1.1947 & -1.1947 & -1.1946 \\
1 & -1.4126 & -1.4189 & -1.4203 & -1.4205 & -1.4212 \\
\hline
\end{tabular}

\begin{tabular}{|c|c|c|c|c|c|c|}
\hline & \multicolumn{2}{|c|}{$\theta=0$} & \multicolumn{2}{|c|}{$\theta=-1 / 60$} & \multicolumn{2}{|c|}{$\theta=-20 / 60$} \\
\hline & Num. & Act. & Num & Act. & Num. & Act \\
\hline$t=0$ & 0 & 0 & -0.0167 & -0.0167 & -0.3000 & -0.3000 \\
\hline $\mathrm{t}=0.1$ & -0.1133 & -0.1142 & -0.0946 & -0.0952 & -0.2000 & -0.2000 \\
\hline $\mathrm{t}=0.2$ & -0.2259 & -0.2278 & -0.2072 & -0.2089 & -0.1029 & -0.1000 \\
\hline $\mathrm{t}=0.3$ & -0.3377 & -0.3406 & -0.3191 & -0.3219 & -0.0599 & -0.0000 \\
\hline $\mathrm{t}=0.4$ & -0.4490 & -0.4527 & -0.4304 & -0.4341 & -0.1209 & -0.1142 \\
\hline $\mathrm{t}=0.5$ & -0.5628 & -0.5639 & -0.5434 & -0.5455 & -0.2262 & -0.2278 \\
\hline $\mathrm{t}=0.6$ & -0.6874 & -0.6853 & -0.6657 & -0.6636 & -0.3376 & -0.3406 \\
\hline $\mathrm{t}=0.7$ & -0.8313 & -0.8293 & -0.8061 & -0.8036 & -0.4495 & -0.4527 \\
\hline $\mathrm{t}=0.8$ & -0.9994 & -0.9982 & -0.9700 & -0.9682 & -0.5652 & -0.5639 \\
\hline $\mathrm{t}=0.9$ & -1.1947 & -1.1946 & -1.1606 & -1.1598 & -0.6920 & -0.6853 \\
\hline $\mathrm{t}=1$ & -1.4205 & -1.4212 & -1.3811 & -1.3812 & -0.8372 & -0.8293 \\
\hline
\end{tabular}

In the tables below, for $n=60$, we compare $v_{i}(t)$ with $x\left(t+\theta_{i}\right)$ for some values of $i$. 


\begin{tabular}{|c|c|c|c|c|c|c|}
\hline & \multicolumn{2}{|c|}{$\theta=-40 / 60$} & \multicolumn{2}{|c|}{$\theta=-1$} & \multicolumn{2}{|c|}{$\theta=-80 / 60$} \\
\hline & Num & Act. & Num. & Act. & Num. & Act. \\
\hline$t=0$ & -0.6667 & -0.6667 & -1.000 & -1.0000 & -1.3333 & -1.3333 \\
\hline$t=0.1$ & -0.5667 & -0.5667 & -0.9000 & -0.9000 & -1.2333 & -1.2333 \\
\hline $\mathrm{t}=0.2$ & -0.4667 & -0.4667 & -0.8000 & -0.8000 & -1.1333 & -1.1333 \\
\hline $\mathrm{t}=0.3$ & -0.3667 & -0.3667 & -0.7000 & -0.7000 & -1.0333 & -1.0333 \\
\hline $\mathrm{t}=0.4$ & -0.2667 & -0.2667 & -0.6000 & -0.6000 & -0.9333 & -0.9333 \\
\hline $\mathrm{t}=0.5$ & -0.1701 & -0.1667 & -0.5000 & -0.5000 & -0.8333 & -0.8333 \\
\hline $\mathrm{t}=0.6$ & -0.1000 & -0.0667 & -0.3999 & -0.4000 & -0.7333 & -0.7333 \\
\hline$t=0.7$ & -0.0975 & -0.0381 & -0.3004 & -0.3000 & -0.6333 & -0.6333 \\
\hline $\mathrm{t}=0.8$ & -0.1642 & -0.1521 & -0.2050 & -0.2000 & -0.5331 & -0.5333 \\
\hline $\mathrm{t}=0.9$ & -0.2650 & -0.2655 & -0.1317 & -0.1000 & -0.4334 & -0.4333 \\
\hline $\mathrm{t}=1$ & -0.3753 & -0.3781 & -0.1096 & -0.0000 & -1.3340 & -0.3333 \\
\hline
\end{tabular}

\begin{tabular}{|c|c|c|c|c|}
\hline & \multicolumn{2}{|c|}{$\theta=-20$} & \multicolumn{2}{|c|}{$\theta=-60$} \\
\hline & Num. & Act & Num & Act. \\
\hline$t=0$ & -20.0000 & -20.0000 & -60.000 & -60.0000 \\
\hline $\mathrm{t}=0.1$ & -19.9000 & -19.9000 & -59.9000 & -59.9000 \\
\hline $\mathrm{t}=0.2$ & -19.8617 & -19.8000 & -59.8000 & -59.8000 \\
\hline $\mathrm{t}=0.3$ & -19.7167 & -19.7000 & -59.7000 & -59.7000 \\
\hline $\mathrm{t}=0.4$ & -19.6167 & -19.6000 & -59.6000 & -59.6000 \\
\hline $\mathrm{t}=0.5$ & -19.5167 & -19.5000 & -59.5000 & -59.5000 \\
\hline $\mathrm{t}=0.6$ & -19.4167 & -19.4000 & -59.4000 & -59.4000 \\
\hline $\mathrm{t}=0.7$ & -19.3167 & -19.3000 & -59.3000 & -59.3000 \\
\hline $\mathrm{t}=0.8$ & -19.2167 & -19.2000 & -59.2000 & -59.2000 \\
\hline $\mathrm{t}=0.9$ & -19.1167 & -19.1000 & -59.1000 & -59.1000 \\
\hline$t=1$ & -19.0167 & -19.0000 & -59.0000 & -59.0000 \\
\hline
\end{tabular}

\section{REFERENCES}

[1] J.K. Hale and J. Kato, Phase space for retarded equations with infinite delay, Funkcial Ekvac., 21 (1978), 11-41. MR0492721(58:11793)

[2] Y. Hino, S. Murakami and T. Naito, Functional-Differential Equations with Infinite Delay, Lecture Notes in Mathematics, 1473, Springer, Berlin, 1991. MR1122588 (92g:34088)

[3] F.V. Atkinson and J.R. Hadddock, On determining phase spaces for functional-differential equations, Funkcial. Ekvac., 31 (1988), 331-347. MR0987790 (90f:45009)

[4] J.R. Haddock, M.N. Nkashama and J. Wu, Asymptotic constancy for linear neutral Volterra integrodifferential equations, Tohuku Math. J., 41 (1989), 689-710. MR1025334(91a:34056)

[5] J. Haddock and J. Terjeki, On the location of positive limit sets for autonomous functionaldifferential equations with infinite delay, J. Diff. Eqns, 86 (1990), 1-31. MR1061887 (91k:34107)

[6] K. Ito and F. Kappel, The Trotter-Kato theorem and approximation of PDEs, Math. Comp. 67 (1998), 21-44. MR1443120 (98e:47060)

[7] T. Sengadir, Semigroups on Frechet Spaces and Equations with Infinite Delays (communicated).

[8] A. Pazy, Semigroups of Linear Operators and Applicaions to Partial Differential Equations, Springer-Verlag, New York, 1983. MR0710486 (85g:47061)

[9] D. Salamon, Structure and stability of finite-dimensional approximations for functionaldifferential equations, SIAM. J. Control and Opt. 23 (1985), 928-951. MR0809542 (87b:34088) 
[10] H.T. Banks and F. Kappel, Spline Approximations for Functional Differential Equations, J. Diff. Eqns. 34 (1979), 496-522. MR.0555324 (81c:65031)

[11] F. Kappel and K. Kunisch, Approximation of the State of Infinite Delay and Volterra-Type Equations, Differential Difference Equations, Applications and Numerical Problems 149-168, Ed. Herausgegeben von, Birkhäuser Verlag, Basel, 1983. MR0726414 (85b:65118)

[12] A. Bellen and M. Zennaro, Numerical methods for delay differential equations, Oxford University Press, Oxford, 2003. MR 1997488 (2004i:65001)

Department of Mathematics, SSN College of Engineering, Old Mahabalipuram Road, KalavakKam-603 110, TAmil Nadu, India 